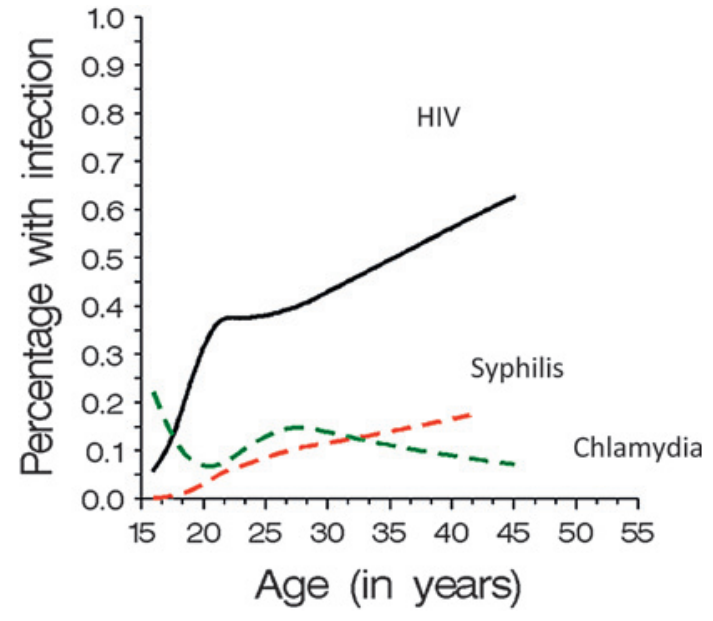

Abstract P1-S2.63 Figure 1

\section{P1-S2.64 DEMOGRAPHIC CHARACTERISTICS AND HIV RISK BEHAVIOURS AMONG MEN WHO HAVE SEX WITH MEN IN GUATEMALA CITY, GUATEMALA, 2010}

doi:10.1136/sextrans-2011-050108.121

${ }^{1} \mathrm{~B}$ Alvarez, ${ }^{1} \mathrm{~W}$ Miller, ${ }^{1} \mathrm{~F} \mathrm{M}$ Hernandez, ${ }^{2} \mathrm{~A}$ Alvarado, ${ }^{1} \mathrm{~S}$ Morales, ${ }^{2} \mathrm{G}$ Paz-Bailey. ${ }^{1}$ Centro de Estudios en Salud, Universidad del Valle de Guatemala, Guatemala; ${ }^{2}$ TEPHINET, Guatemala

Background In Central America, surveillance of HIV has been strengthened by regular and systematic collection of HIV, sexually transmitted infection, and behavioural data among most at risk populations. In Guatemala, men who have sex with men (MSM) are a highly vulnerable to HIV infection. Behavioural data are needed to help plan for prevention interventions in this group.

Methods Male residents of Guatemala City at least 18 years of age who self-reported having had anal sex with another man in the past 12 months were invited to participate in a face-to-face structured behavioural survey using respondent-driven-sampling (RDS) from September to December 2010. Participants who provided informed consent were administered a questionnaire that collected information on socio-demographics, risk behaviour, knowledge and attitudes, access to services, and stigma and discrimination, using personal digital assistants (PDA). Medians and IORs were calculated for continuous variables. Population proportions were calculated for categorical variables and adjusted for RDS-sampling weights using Respondent Driven Sampling Analysis Tool version 6.0 .

Results A total of 500 MSM enrolled in the study. Respondents self-identified as gay or homosexual (39.5\%), bisexual $(39.5 \%)$, heterosexual (11.5\%), and transvestite or transgender $(9.5 \%)$. Median age was 26 years (IOR 22-34 years). The majority of respondents $(79.5 \%)$ reported to have access to free condoms in the past 12 months. Consistent condom use with all male partners in last 12 months and during last sex was reported by $62.2 \%$ and $75.4 \%$, respectively. Overall, $44.3 \%$ reported having sex with a woman in the past 12 months. Consistent condom use with female sexual partners in the last 12 months and during last sex was reported by $46.1 \%$ and $66.0 \%$, respectively. The median number of female sexual partners reported in the last 12 months was 3 (IOR: 2-5). Ever paying for sex and paying for sex in the last 12 months was reported by $16.0 \%$ and $8.3 \%$, respectively; while $54.0 \%$ and $44.7 \%$ reported ever selling sex and in the last 12 months, respectively. Injection drug use in the last 12 months was reported by $0.8 \%$. Overall, $73.3 \%$ of respondents reported ever having an HIV test and $43.1 \%$ had been tested in the past 12 months.

Conclusions MSM in Guatemala engage in high risk behaviours that place them and their male and female partners at high risk for HIV infection. Innovative strategies are needed to promote condom use and access to HIV testing and counselling.

\section{P1-S2.65 CRACK/COCAINE USE AMONG MSM IN LATIN AMERICA: A MULTILEVEL ANALYSIS OF RDS STUDIES TO IDENTIFY DRUG USE PATTERNS AND ASSOCIATED FACTORS ACROSS CITIES}

doi:10.1136/sextrans-2011-050108.122

${ }^{1} \mathrm{~J} O$ Jacobson, ${ }^{1} \mathrm{M}$ Alonso-Gonzalez, ${ }^{2} \mathrm{D}$ Ramachandran, ${ }^{3} \mathrm{~S}$ Morales-Miranda, ${ }^{4}$ A Carballo-Dieguez, ${ }^{5} \mathrm{~J}$ Medrano, ${ }^{6} \mathrm{~T}$ Solano, ${ }^{1} \mathrm{M} \mathrm{D}$ Rosales-Perez. ${ }^{1}$ Pan American Health Organization, Bogota, Colombia; ${ }^{2} J o h n s$ Hopkins Bloomberg School of Public Health, Baltimore, USA; ${ }^{3}$ Del Valle University of Guatemala, Guatemala City, Guatemala; ${ }^{4}$ Columbia University, New York City, USA; ${ }^{5}$ Ministerio de Salud y Deportes, La Paz, Bolivia; ${ }^{6}$ Ministerio de Salud de Costa Rica, San Jose, Costa Rica

Background In Latin America (LA), men who have sex with men (MSM) are the population most affected by HIV, crack/cocaine production and trafficking routes are well-established, and local consumption has risen sharply in recent years. Yet, drug use and associations with sexual risk behaviours among MSM remain largely unexamined LA despite being recognised as important drivers of HIV transmission in the USA. Variation in crack/cocaine consumption within LA is also unknown.

Methods Data from participants self-identifying as MSM and aged 18 years and older in recent (2006-2009) respondent-driven sampling (RDS) studies using similar survey methodologies in nine high-population cities in Argentina, Bolivia, Costa Rica, El Salvador and Honduras $(\mathrm{N}=2081)$ were pooled and analysed in a multilevel statistical framework to increase statistical power to evaluate past 12-month crack/cocaine consumption (CC). CC prevalence was estimated by study site, adjusting for sampling weights incorporating personal network and population sizes. Logistic, multilevel models identified correlates of CC, adjusted for intraclass correlation within levels defined by study site and recruitment chain, and partitioned variance in CC among levels.

Results CC prevalence was $21.7 \%$ (95\% CI 17.4 to 26.7 ) in the pooled sample and varied significantly across cities from $0.1 \%$ in Cochabamba, Bolivia to $44.5 \%$ in Ceiba, Honduras. In multivariate analysis, daily alcohol consumption (adjusted OR $[\mathrm{AOR}]=4.0$ ), sexual debut of $\leq 12(\mathrm{AOR}=3.1)$ and $13-17(\mathrm{AOR}=1.9)$ years old, self-identifying as transexual/transgender/transvestite (TTT) $(\mathrm{AOR}=2.2)$, age $18-22$ ( $\mathrm{vs}>40$ ) years $(\mathrm{AOR}=2.0)$, having a stable female partner $(A O R=1.8)$ and ever purchasing sex $(A O R=1.6)$ were associated with CC $(p<0.05)$. Significant city-level random effects expressed as $\mathrm{AOR}$ were $5.4(\mathrm{SE}=1.3)$ and $2.7(\mathrm{SE}=1.3)$ in Ceiba and San Pedro Sula, Honduras, respectively and $3.4(\mathrm{SE}=1.2)$ in Buenos Aires, Argentina. Of the total variance in CC, $29.3 \%$ was associated with unobserved differences among cities. Associations between CC and condom use at last sex, and recruitment chain, were nonsignificant $(\mathrm{p}>0.1)$.

Conclusions CC prevalence among MSM, and variation among cities in LA, are considerable. Associations with frequent drinking and sexual risk Behaviours likely compound HIV transmission risk among MSM and to female partners. Substance abuse prevention and treatment for MSM are needed, particularly among TTT, and in Honduras and Argentina. 